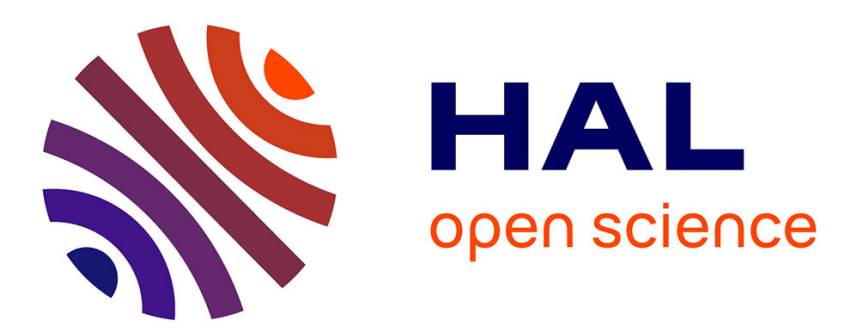

\title{
Three-Dimensional Motion Estimation of Atmospheric Layers From Image Sequences
}

\author{
Patrick Héas, Etienne Mémin
}

\section{To cite this version:}

Patrick Héas, Etienne Mémin. Three-Dimensional Motion Estimation of Atmospheric Layers From Image Sequences. IEEE Transactions on Geoscience and Remote Sensing, 2008, 46 (8), pp.2385-2396. 10.1109/TGRS.2008.918167 . hal-00670348

\section{HAL Id: hal-00670348 \\ https://hal.inria.fr/hal-00670348}

Submitted on 15 Feb 2012

HAL is a multi-disciplinary open access archive for the deposit and dissemination of scientific research documents, whether they are published or not. The documents may come from teaching and research institutions in France or abroad, or from public or private research centers.
L'archive ouverte pluridisciplinaire HAL, est destinée au dépôt et à la diffusion de documents scientifiques de niveau recherche, publiés ou non, émanant des établissements d'enseignement et de recherche français ou étrangers, des laboratoires publics ou privés. 


\title{
3D motion estimation of atmospheric layers from image sequences
}

\author{
Patrick Héas and Etienne Mémin \\ INRIA/IRISA, Rennes, France, \\ \{Patrick.Heas, Etienne.Memin\}@irisa.fr
}

\begin{abstract}
In this paper, we address the problem of estimating three-dimensional motions of a stratified atmosphere from satellite image sequences. The analysis of three-dimensional atmospheric fluid flows associated with incomplete observation of atmospheric layers due to the sparsity of cloud systems is very difficult. This makes the estimation of dense atmospheric motion field from satellite images sequences very difficult. The recovery of the vertical component of fluid motion from a monocular sequence of image observations is a very challenging problem for which no solution exists in the literature. Based on a physically sound vertical decomposition of the atmosphere into cloud layers of different altitudes, we propose here a dense motion estimator dedicated to the extraction of three-dimensional wind fields characterizing the dynamics of a layered atmosphere. Wind estimation is performed over the complete three-dimensional space using a multi-layer model describing a stack of dynamic horizontal layers of evolving thickness, interacting at their boundaries via vertical winds. The efficiency of our approach is demonstrated on synthetic and real sequences.
\end{abstract}

\section{INTRODUCTION}

Geophysical motion characterization and analysis by image sequence analysis is a crucial issue for numerous scientific domains involved in the study of climate change, weather forecasting, climate prediction or biosphere analysis. The use of surface station, balloon, and more recently in-flight aircraft measurements and satellite images has improved the estimation of wind fields and has been a subsequent step towards a better understanding of meteorological phenomena. However, the network's temporal and spatial resolutions may be insufficient for the analysis of mesoscale dynamics. Recently, in an effort to avoid these limitations, another generation of satellites sensors has been designed, providing image sequences characterized by finer spatial and temporal resolutions. Nevertheless, the analysis of motion remains particularly challenging due to the complexity of atmospheric dynamics at such scales.

Tools are needed to exploit this new generation of satellite images and we believe that it is very important that the computer vision community gets involved in such domain as they can potentially bring relevant contributions with respect to the analysis of spatio-temporal data.

Nevertheless in the context of geophysical motion analysis, standard techniques from Computer Vision, originally designed for bi-dimensional quasi-rigid motions with stable salient features, appear to be not well adapted [2] [9] [13] [15]
[19] [20] [22]. The design of techniques dedicated to fluid flow has been a step forward, towards the constitution of reliable methods to extract characteristic features of flows [5] [6] [10] [17] [23] [24]. However, for geophysical applications, existing fluid-dedicated methods are all limited to horizontal velocity estimation and neglect vertical motion. All these methods are obviously not adapted to the extraction of 3D measurements but also do not take into account accurately luminance variations due to $3 \mathrm{D}$ motions. Such effects are occasionally important at mesoscales systems such as convective towers and should be incorporated in the motion estimation method.

Geophysical flows are quite well described by appropriate physical models. As a consequence in such contexts, a physically-based approach can be very powerful for analyzing incomplete and noisy image data, in comparison to standard statistical methods. The inclusion of physical a priori leads to novel advanced techniques for motion analysis or 3D information recovery. This yields to new application domains impacting potentially studies of capital interest for our everyday life, and obviously to the devise of proper efficient techniques. This is thus a research domain with wide perspectives. Our work is a contribution towards this direction.

The method proposed in this paper is significantly different from previous works on motion analysis by satellite imagery. A main difference is that the 3D data model used in our method relies on a physical model for a stack of pressure difference image observations retrieved at different atmospheric levels. This interacting layered model allows us to recover vertical motion information.

\section{RELATED WORKS ON OPTICAL FLOW ESTIMATION}

The problem of wind field recovery consists in estimating the 3D atmospheric motion denoted by $\mathbf{V}(\mathbf{s}, t)$ from a 2D image sequence $I(\mathbf{s}, t)$, where $(\mathbf{s}, t)$ denote the pixel and time coordinates. This problem is a complex one, for which we have only access to projected information on clouds position and spectral signatures provided by satellite observation channels. To avoid the three-dimensional wind field reconstruction problem, all developed methods have relied on the assumption of negligible vertical winds and focused on the estimation of horizontal winds related to the 
top of clouds which may be at different heights.

Let us present a brief survey on existing models for horizontal motion estimation from image sequences. The estimation of the apparent motion $\mathbf{v}(\mathbf{s}, t)$ as perceived through image intensity variations (the so-called optical-flow) relies principally on the temporal conservation of some invariants. The most common invariant used is the brightness consistency assumption. This assumption leads to the well known OpticalFlow Constraint (OFC) equation

$$
\mathbf{v} \cdot \nabla I(\mathbf{s}, t)+I_{t}(\mathbf{s}, t)=0 .
$$

An important remark is that for image sequences showing evolving atmospheric phenomena, the brightness consistency assumption does not properly model temporal distortions of luminance patterns caused by 3D flow. In spite of this, most estimation methods used in the meteorology community still rely on this crude assumption [14] [19] [20]. The Integrated Continuity Equation (ICE) provides a valid invariant assumption for altimetric imagery [7] of incompressible flows or for transmittance imagery of compressible flows [8] under the assumption that the temporal derivatives of the integration boundaries compensate the normal flows. This ICE model reads :

$$
\left(\int \rho d z\right)_{t}+\mathbf{v} \cdot \nabla\left(\int \rho d z\right)+\left(\int \rho d z\right) \operatorname{div} \mathbf{v}=0
$$

where $\rho$ and $\mathbf{v}$ denote the fluid density and the density averaged horizontal motion field along the vertical axis. Unlike the OFC, such models can compensate mass departures observed in the image plane by associating two-dimensional divergence to brightness variations. But, for the case of satellite infra-red imagery, the assumption that $I \propto \int \rho d z$ is flawed. Moreover, note that although the assumed boundary condition is valid for incompressible flows, it is not realistic for compressible atmospheric flows observed at a kilometer order scale. However, based on experiments, the authors proposed to apply this model directly to the image infra-red observations [5] or to the inverse of the image infra-red intensities [24]. Such models, although inexact for infra-red images, have shown to provide better results than a data model based on brightness consistency.

Recently, under the assumption of negligible vertical wind (which makes valid the assumption that the temporal derivatives of the surface corresponding to integration boundaries compensate the normal flows), the model of Eq. 2 has been applied to pressure difference maps approximating the density integrals [10].

The formulations of Eq.1 and Eq.2 can not be used alone, as they provide only one equation for two unknowns at each spatio-temporal locations $(\mathbf{s}, t)$, with therefore, a one dimensional family of solutions in general. In order to remove this ambiguity and improve the robustness of the estimation, the most common assumption is to reinforce the spatial local coherence. This local coherence can explicitly be formalized as a regularity prior within a globalized smoothing scheme. Within this scheme, the spatial dependencies are modeled on the complete image domain. As a result, the scheme is more robust in its ability to handle noisy and low contrast observations. More precisely, the motion estimation problem is defined as the global minimization of an energy function composed of two components :

$$
J(\mathbf{v}, I)=J_{d}(\mathbf{v}, I)+\alpha J_{r}(\mathbf{v}) .
$$

The first component $J_{d}(\mathbf{v}, I)$ called the data term, expresses the constraint linking unknowns to observations while the second component $J_{r}(\mathbf{v})$, called the smoothing term, reinforce the solution to follow some smoothness properties. The parameter, $\alpha$, controls the balance between the smoothness and the global adequacy to the observation model. In this framework, Horn and Schunck [13] first introduced a data term related to the OFC equation and a first-order smoothing of the two spatial components $u$ and $v$ of velocity field $\mathbf{v}$. In the case of transmittance imagery of fluid flows, $I=\int \rho d z$, and using the previously defined ICE model (Eq.2) leads to the functional :

$$
J_{d}(\mathbf{v}, I)=\int_{\Omega}\left(I_{t}(\mathbf{s})+\mathbf{v}(\mathbf{s}) \cdot \nabla I(\mathbf{s})+I(\mathbf{s}) \operatorname{div} \mathbf{v}(\mathbf{s})\right)^{2} d \mathbf{s},
$$

where $\Omega$ denotes the image domain. Recently, a dedicated method has been proposed to solve a multi-layered motion estimation problem by the inference of dense density-weighted average horizontal wind fields related to different atmospheric strata [10]. In this method, the motion extraction is done by fitting an image-adapted transmittance conservation model (i.e. the ICE model of Eq. 2) to pressure observations, independently for the different layers. To cope with the complexity of atmospheric motion observed at mesoscale and with noisy and sparse observations, a two-stage estimation scheme has been introduced. It incorporates correlationbased constraints and a priori information on atmospheric dynamics [10].

It can be demonstrated that a first order smoothing is not adapted as it favors the estimation of velocity fields with low divergence and low vorticity. A second order smoothing on the vorticity and the divergence of the defined motion field can advantageously be considered as proposed in [5] [21] [23] :

$$
J_{r}(\mathbf{v})=\int_{\Omega}\|\nabla \operatorname{curl} \mathbf{v}(\mathbf{s})\|^{2}+\|\nabla \operatorname{div} \mathbf{v}(\mathbf{s})\|^{2} d \mathbf{s} .
$$

Instead of relying on a $\boldsymbol{L}^{2}$ norm, a robust penalty function $\phi_{d}$ may be introduced in the data term for attenuating the effect of observations deviating significantly from the ICE constraint [4]. Similarly, a robust penalty function $\phi_{r}$ can be used if one wants to handle implicitly the spatial discontinuities of the vorticity and divergence maps. In the image plane, these discontinuities are nevertheless difficult to relate to abrupt variations of clouds height. Moreover, the robust approach does not allow points of unconnected regions, which belong to a same layer, to interact during the motion estimation process. 


\section{MOdELING A DYNAMiCAL STACK OF INTERACTING} LAYERS

In this section, we revisit the integrated continuity equation expressed in a pressure coordinate system in the presence of vertical winds in order to derive a 3D dynamical model for a stack of interacting layers fitting sparse pressure difference observations. This atmospheric model extends to $3 \mathrm{D}$, the horizontal mass conservation model proposed in [10].

\section{A. Revisiting the Integrated Continuity Equation for 3D winds}

Interesting models for 3D compressible atmospheric motion observed through image sequences may be derived by integrating the $3 \mathrm{D}$ continuity equation expressed in the isobaric coordinate system $(x, y, p)$. In comparison to standard altimetric coordinates, isobaric coordinates are advantageous : they enable to handle in a simple manner the compressibility of atmospheric flows while dealing directly with pressure quantities, which will be used as observations in this paper. In this coordinate system, the pressure function $p$ acts as a vertical coordinate. Let us denote the horizontal wind components by $\mathbf{v}=(u, v)$ and the vertical wind in isobaric coordinates by $\omega$. The 3D continuity equation reads [12] :

$$
-\frac{\partial \omega}{\partial p}=\left(\frac{\partial u}{\partial x}+\frac{\partial v}{\partial y}\right)_{p} .
$$

By defining now two altimetric surfaces $s^{k}$ and $s^{k+1}$ with $p\left(s^{k}\right)>p\left(s^{k+1}\right)$ related to a pressure difference function $\delta p^{k}$ and a pressure-average horizontal wind field $\mathbf{v}^{k}$

$$
\begin{aligned}
\delta p^{k} & =p\left(s^{k}\right)-p\left(s^{k+1}\right) \\
\mathbf{v}^{k} & =\frac{1}{\delta p^{k}} \int_{p\left(s^{k+1}\right)}^{p\left(s^{k}\right)} \mathbf{v} d p,
\end{aligned}
$$

we have demonstrated in appendix I that, under certain conditions, the vertical integration of Eq. 6 in the altimetric interval $\left[s^{k}, s^{k+1}\right]$ yields to the following 3D-ICE model:

$$
g \rho\left(s^{k}\right) \mathrm{w}\left(s^{k}\right)-g \rho\left(s^{k+1}\right) \mathrm{w}\left(s^{k+1}\right)=\frac{d \delta p^{k}}{d t}+\delta p^{k} \operatorname{div}\left(\mathbf{v}^{k}\right),
$$

where $g$ and w denote respectively the gravity constant and the vertical wind in the standard altimetric coordinate system $(x, y, z)$. Note that this model appears to be a generalization of the so called kinematic method applied in meteorology for the recovery of vertical motion [12]. Indeed, by neglecting the first term on the right hand side of Eq.9, vertical motion can be expressed as :

$$
\mathrm{w}\left(s^{k+1}\right)=\frac{\rho\left(s^{k}\right) \mathrm{w}\left(s^{k}\right)}{\rho\left(s^{k+1}\right)}-\frac{\delta p^{k}}{g \rho\left(s^{k+1}\right)} \operatorname{div}\left(\mathbf{v}^{k}\right),
$$

which corresponds exactly to the kinematic estimate. Note also that the ICE model (Eq.2) used in [10] can be recovered when vertical motion is neglected and for an atmosphere in hydrostatic equilibrium ( $\left.\delta p=-g \int \rho d z\right)$. On the right side of the $3 \mathrm{D}-\mathrm{ICE}$, vertical motion $\mathrm{w}$ appears only on the integration boundaries, while on the left side, pressure-average horizontal motion $\mathbf{v}^{k}$ appears within a standard optical flow expression compensated by a divergence correcting term. Thus, for pressure difference observations on layer boundaries, the 3D-ICE constitutes a possible 3D estimation model.

\section{B. Layer decomposition}

The layering of atmospheric flow in the troposphere is valid in the limit of horizontal scales much greater than the vertical scale height, thus roughly for horizontal scales greater than $100 \mathrm{~km}$. It is thus impossible to truly characterize a layered atmosphere with a local analysis performed in the vicinity of a pixel characterizing a kilometer order scale. Nevertheless, one can still decompose the 3D space into elements of variable thickness, where only sufficiently thin regions of such elements may really correspond to common layers. Analysis based on such a decomposition presents the main advantage of operating at different atmospheric pressure ranges and avoids the mix of heterogeneous observations.

For the definition of layers, we present the 3D space decomposition introduced in [10]. The $k$-th layer corresponds to the volume lying in between an upper surface $s^{k+1}$ and a lower surface $s^{k}$. These surfaces $s^{k}$ are defined by the height of top of clouds belonging to the $k$-th layer. They are thus defined only in areas where there exists clouds belonging to the $k$-th layer, and remains undefined elsewhere. The membership of top of clouds to the different layers is determined by cloud classification maps. Such classifications, which are based on thresholds of top of cloud pressure, are routinely provided by the EUMETSAT consortium, the European agency which supplies the METEOSAT satellite data. Note that the discrimination of layers according to classifications based on top of cloud potential temperature rather than top of cloud pressure would have been better suited to mesoscale. However, such classification are not currently available.

\section{Sparse pressure difference observations}

In order to derive pressure difference observations at different atmospheric levels, we rely on top of cloud pressure images as proposed in [10]. Indeed, top of cloud pressure images are also routinely provided by the EUMETSAT consortium. They are derived from a radiative transfer model using ancillary data obtained by analysis or short term forecasts. This model simulates the radiation at the top of an opaque cloud at different vertical levels, which might be observed by a satellite. The pressure level where the simulated radiation fits best with the observed radiation determines the pressure of the cloud top for the corresponding pixel [16]. Multi-channel techniques (using a thermal IR with a water vapor or $\mathrm{CO}_{2}$ absorption channels) enable the determination of the temperature of the top of semi-transparent clouds [18] [20], and thus their equivalent pressure level, with the help of analyzed or forecast data.

We denote by $C^{k}$ the class corresponding to the $k$-th layer. Note that the top of cloud pressure image denoted by $p_{\cup_{k}}$ is composed of segments of top of cloud pressure functions $p\left(s^{k+1}\right)$ related to the different layers. That is to say $: p_{\cup_{k}}=$ $\left\{\bigcup_{k} p\left(s^{k+1}, \mathbf{s}\right) ; \mathbf{s} \in C^{k}\right\}$. Thus, pressure images of top of clouds are used to constitute sparse pressure maps of the layer upper boundaries $p\left(s^{k+1}\right)$. Since cloud bases are not readily observed in satellite imagery, we coarsely approximate the 
missing pressure observations $p\left(s^{k}\right)$ by an average pressure value $\bar{p}^{k}$ observed on top of clouds of the layer underneath. Finally, for the $k$-th layer, we define observations $h^{k}$ as pressure differences :

$$
\bar{p}^{k}-p_{\cup_{k}}=h^{k}\left\{\begin{array}{lll}
=\delta p^{k}(\mathbf{s}) & \text { if } & \mathbf{s} \in C^{k} \\
\neq \delta p^{k}(\mathbf{s}) & \text { if } & \mathbf{s} \in \bar{C}^{k}
\end{array}\right.
$$

\section{Layer interacting model}

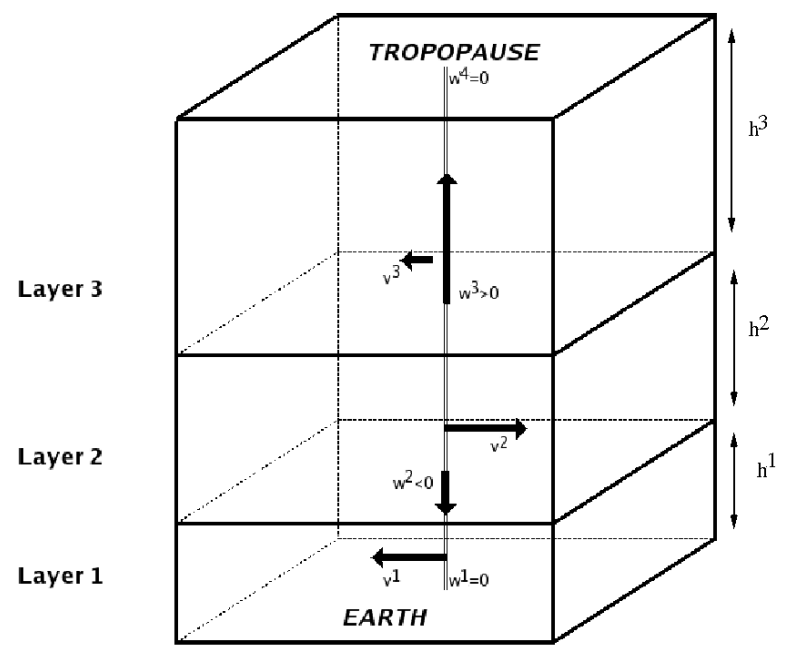

Fig. 1. Scheme of three interacting layers defined at a given pixel location. The set of unknowns associated to the corresponding 3D-ICE model is $\left\{\mathbf{v}^{1}, w^{2}, \mathbf{v}^{2}, w^{3}, \mathbf{v}^{3}\right\}$. For the enhancement of the visual representation, pressure difference $h^{k}$ have been identified here to altimetric heights.

Eq.9 is thus valid for image observations $h^{k}$ related to the $k$-th layer on the spatial sub-domain $C^{k}$ :

$$
\frac{d\left(h^{k}\right)}{d t}+h^{k} \nabla \cdot \mathbf{v}^{k}=g\left(\rho^{k} \mathbf{w}^{k}-\rho^{k+1} \mathbf{w}^{k+1}\right),
$$

where for clarity we have simplified notations $\rho\left(s^{k}\right)$ and $\mathrm{w}\left(s^{k}\right)$ into $\rho^{k}$ and $\mathrm{w}^{k}$. As density fields on the layer surfaces are unknown, we simplify the modeling by assuming that density fields $\rho^{k}$ are constants with values $\bar{\rho}^{k}$. Such values can roughly be related to the average pressures $\bar{p}^{k}$ by vertical integration of the equation of state for dry air $(p=\rho R T)$ combined with the hydrostatic relation $\left(d p=-g \int \rho d z\right)$ under the assumption of constant lapse rate $\left(T=T_{0}+\gamma z\right)$ where $R, T_{0}$ and $\gamma$ denote physical constants. More precisely, between a reference altitude $z_{0}$ (or pressure $p_{0}$ ) and an average altitude $\bar{z}^{k}$ (or pressure $\bar{p}^{k}$ ), one obtains the relation :

$$
\int_{p_{0}}^{\bar{p}^{k}} \frac{d p}{p} \approx-\int_{z_{0}}^{\bar{z}^{k}} \frac{g}{R\left(T_{0}+\gamma z\right)} d z
$$

which yields after some calculation to the coarse approximation :

$$
\bar{\rho}^{k} \approx \frac{p_{0}}{R T_{0}}{\frac{\bar{p}^{k}}{p_{0}}}^{\frac{\gamma R}{g}+1} .
$$

Although the constant lapse rate assumption, that is to say the linear variation of temperature with altitude, is valid in the troposphere only to some extent, note that in the model of Eq. 12, density horizontal fluctuations may be annihilated by vertical wind errors. And, performing a scale analysis in a pixel vicinity, it can be shown that such fluctuations are too weak (magnitude of $10^{-2} \mathrm{~s}^{2} \mathrm{~m}^{-2} \mathrm{~Pa}^{-1}$ ) in comparison to the density amplitude (magnitude of $1 \mathrm{~s}^{2} \mathrm{~m}^{-2} \mathrm{~Pa}^{-1}$ ) to have a significant impact on vertical wind amplitude (magnitude of $10^{-1} \mathrm{~ms}^{-1}$ ) [12].

After integrating in time the differential equation 12 along the horizontal trajectories and applying the variation of the constant tecnhique for the second member, we obtain a timeintegrated form :

$$
\begin{aligned}
\text { if } \operatorname{div}^{k} \neq 0 & : \\
\tilde{h}^{k} e^{\operatorname{div} \mathbf{v}^{k}}-h^{k} & =g \Delta t \frac{\bar{\rho}^{k} \mathrm{w}^{k}-\bar{\rho}^{k+1} \mathrm{w}^{k+1}}{\operatorname{div} \mathbf{v}^{k}}\left(e^{\operatorname{div} \mathbf{v}^{k}}-1\right), \\
\text { if } \operatorname{div} \mathbf{v}^{k}=0 & : \\
\tilde{h}^{k}-h^{k} & =-g \Delta t\left(\bar{\rho}^{k} \mathrm{w}^{k}-\bar{\rho}^{k+1} \mathrm{w}^{k+1}\right) .
\end{aligned}
$$

where the motion-compensated image $h^{k}\left(\mathbf{s}+\mathbf{v}^{k}, t+\Delta t\right)$ has been denoted for convenience by $\tilde{h}^{k}$ and where $\Delta t$ denotes the time interval expressed in seconds between two consecutive images. Details on the derivation of the time-integrated form are provided in appendix II.

For the lowest layer, the Earth boundary condition implies : $\mathrm{w}^{1}=0$. Let $K$ denote the index of the highest layer. Another boundary conditions may be given for the highest layer by the reasonable assumption that vertical wind can be neglected at the tropopause which acts like a cover : $\mathrm{w}^{K+1}=$ 0 . Thus, as the vertical wind present on the upper bound of the $k$-th layer is identical to the one present on the lower bound of the $(k+1)$-th layer, we have the following two sets of unknowns : $\left\{v^{k}: k \in[1, K]\right\}$ and $\left\{\mathrm{w}^{k}: k \in[2, K]\right\}$. The vertical wind unknowns act as variables that express horizontal wind interactions between adjacent layers. Fig. 1 schematizes an example of three interacting layers associated to a set of unknowns, according to the 3D-ICE model.

\section{3D WIND ESTIMATION}

In this section, we present a robust estimator based on the layer interacting model introduced previously. This method extends to $3 \mathrm{D}$ the layered horizontal motion estimation proposed in [10].

\section{A. Dedicated robust estimator}

Since outside the class $C^{k}, h^{k}$ defined in Eq.11 is not relevant of the $k$-th layer, we introduce a masking operator to remove unreliable observations by saturation of a robust penalty function $\phi_{d}$. More explicitly, we denote by $\mathbb{I}_{C^{k}}$ the operator which is identity if pixel belong to the class, and which returns a fixed value out of the range taken by $h^{k}$ otherwise. Thus, applying this new masking operator in Eq.15, we obtain for the $k$-th layer the robust data term $J_{d}\left(\mathbf{v}^{k}, \mathrm{w}^{k}, \mathrm{w}^{k+1}, h^{k}\right)=$ 


$$
\begin{aligned}
& \text { if }\left\|\operatorname{divv}^{k}(\mathbf{s})\right\| \geq \epsilon: \\
& \int_{\Omega} \phi_{d}\left[\tilde{h}^{k}(\mathbf{s}) \exp \left\{\operatorname{divv}^{k}(\mathbf{s})\right\}-\mathbb{I}_{C^{k}}\left(h^{k}(\mathbf{s})\right)\right. \\
& \left.+g \Delta t \frac{\bar{\rho}^{k} \mathbf{w}^{k}(\mathbf{s})-\bar{\rho}^{k+1} \mathbf{w}^{k+1}(\mathbf{s})}{\operatorname{divv}^{k}(\mathbf{s})}\left(1-\exp \left\{\operatorname{divv}^{k}(\mathbf{s})\right\}\right)\right] d \mathbf{s} \\
& \text { else : } \\
& \int_{\Omega} \phi_{d}\left[\tilde{h}^{k}(\mathbf{s})-\mathbb{I}_{C^{k}}\left(h^{k}(\mathbf{s})\right)\right. \\
& \left.-g \Delta t\left(\bar{\rho}^{k} \mathbf{w}^{k}(\mathbf{s})-\bar{\rho}^{k+1} \mathbf{w}^{k+1}(\mathbf{s})\right)\right] d \mathbf{s},
\end{aligned}
$$

where $\epsilon$ is a scalar close to zero. A second order div-curl smoother has been chosen to constrain spatial smoothness of horizontal wind fields. The latter was combined with a first order smoother that reinforces regions of homogeneous vertical winds. Note that we have restricted the smoother for vertical wind to be a first order one, as 3D divergence and $3 \mathrm{D}$ vorticity vectors are inaccessible in a layered model. The smoothing term for the $k$-th layer has been thus defined as $J_{r}\left(\mathbf{v}^{k}, \mathrm{w}^{k}\right)=$

$\int_{\Omega} \alpha\left(\left\|\nabla \operatorname{curl} \mathbf{v}^{k}(\mathbf{s})\right\|^{2}+\left\|\nabla \operatorname{divv}^{k}(\mathbf{s})\right\|^{2}\right)+\beta\left\|\nabla \mathbf{w}^{k}(\mathbf{s})\right\|^{2} d \mathbf{s}$,

where $\beta>0$ denotes a positive parameter. A Leclerc Mestimator has been chosen for $\phi_{d}$ for its advantageous minimization properties [11]. The masking procedure, together with the use of this robust penalty function on the data term, allows erroneous observations from the estimation process to be discarded. It is important to note that, for the $k$-th layer, the method provides estimates on all point $s$ of the image domain $\Omega$. Areas outside the cloud class $C^{k}$ correspond to $3 \mathrm{D}$ interpolated wind fields.

\section{B. Large horizontal displacements}

One major problem with the differential formulation of Eq.12 is the estimation of large displacements. However, the integrated form of Eq.15 is valid for high amplitude displacements, and has the advantage of being linear. A standard approach for tackling the non-linear data term is to apply successive linearizations around a current estimate and to warp a multi-resolution representation of the data accordingly. This approach relies on an image pyramid, constructed by successive low-pass filtering and down sampling of the original images. A large displacement field $\tilde{\mathbf{v}}$ is first estimated at coarse resolution where motion amplitude should be sufficiently reduced in order to make the initial differential data model valid. Then, the estimation is refined through an incremental fields $\mathbf{v}^{\prime}$ while going down the pyramid [3]. The latter are estimated within a linear scheme by minimizing linearized motion-compensated functionals : for the decomposition $\mathbf{v}^{k}=$ $\tilde{\mathbf{v}}+\mathbf{v}^{\prime}$, Eq.16 is linearized around $\tilde{\mathbf{v}}$ and yields to a motioncompensated linear formulation of the data term. Let us denote by $\tilde{\zeta}^{k}$ the coarse scale divergence estimate $\operatorname{div} \tilde{\mathbf{v}}$ and omit for sake of clarity point coordinates $\mathbf{s}$ in the integrals. After some calculation, we obtain that, for the $k$-th layer, the linearized data term reads $J_{d}\left(\mathbf{v}^{k}, \mathrm{w}^{k}, \mathrm{w}^{k+1}, h^{k}\right)=$

$$
\begin{array}{r}
\int_{\Omega} \phi_{d}\left\{e^{\tilde{\zeta}^{k}}\left(\left[\tilde{h}^{k} \nabla \tilde{\zeta}^{k}+\nabla \tilde{h}^{k}\right]^{T} \mathbf{v}^{\prime}+\tilde{h}^{k}\right)-\mathbb{I}_{C^{k}}\left(h^{k}\right)\right. \\
\left.+g \Delta t f\left(\tilde{\zeta}^{k}, \mathbf{w}^{k}, \mathbf{w}^{k+1}\right)\right\} d \mathbf{s}
\end{array}
$$

where if $\left\|\tilde{\zeta}^{k}\right\| \geq \epsilon, f\left(\tilde{\zeta}^{k}, \mathrm{w}^{k}, \mathrm{w}^{k+1}\right)=$

$$
\frac{\bar{\rho}^{k} \mathrm{w}^{k}-\bar{\rho}^{k+1} \mathrm{w}^{k+1}}{\tilde{\zeta}^{k}}\left(1-e^{\tilde{\zeta}^{k}}+\mathbf{v}^{\prime} \nabla \tilde{\zeta}^{k}\left(\frac{e^{\tilde{\zeta}^{k}}-1}{\tilde{\zeta}^{k}}-e^{\tilde{\zeta}^{k}}\right)\right)
$$

else, $\quad f\left(\tilde{\zeta}^{k}, \mathrm{w}^{k}, \mathrm{w}^{k+1}\right)=\bar{\rho}^{k+1} \mathrm{w}^{k+1}-\rho^{k} \mathrm{w}^{k}$.

In order to enhance the estimation accuracy, a two-stage estimation scheme including correlation-based constraints and a priori information on mesoscale atmosphere dynamics can constitute an alternative approach to common multi-resolution [10]. In the first stage of such an estimation scheme, the vertical motion component is neglected as the estimation performs at large scales (of order of $100 \mathrm{~km}$ ). As a matter of fact, in this stage, large scale displacement estimation relies on the (2D) ICE model. Moreover, large displacements are constrained by a collection of correlation-based vectors and a sound temporal smoother. In the second refinement stage acting at fine scale (of order of $1 \mathrm{~km}$ ), the correlation-based constraints together with the temporal smoother are disconnected and the (2D) ICE model is replaced by the 3D ICE model. This second stage implies the use of the motion-compensated expression of Eq. 18.

\section{Minimization issues}

In the proposed optimization scheme, we chose to minimize a discretize version of functionals of Eq. 18 and Eq. 17. Let us denote by $z^{k}$ the robust weights associated to the semiquadratic penalty function related to the data term. Minimization is done by alternatively solving large systems for unknowns $\mathbf{v}^{k}, \mathrm{w}^{k}$ and $z^{k}$ through a multigrid Gauss-Seidel solver. More explicitly, all variables are first initialized to zero. A global optimization procedure is then successively operated at each level of the multi-resolution pyramid. This procedure first performs in a multigrid optimization strategy, the minimization with respect to $\mathbf{v}^{k}$ of a linearized functional composed of the data term defined in Eq.18 and of the second order smoothness term defined in Eq.17. As variables $\left\{\mathrm{w}^{k}\right\}$ and $\left\{z^{k}\right\}$ are first frozen, this first step can be performed independently for each layer level $k \in[1, K]$. Once the minima have been reached, in a second step, fixing variables $\left\{\mathbf{v}^{k}\right\}$ and $\left\{z^{k}\right\}$, the same functional is minimized with respect to each $\mathrm{w}^{k}, k \in[2, K]$. For a neighboring system $\nu_{\mathrm{s}}$, the linear system can be solved iteratively. At the $n$-th iteration, the Gauss-Seidel update for vertical wind is expressed for each pixel location $\mathbf{s} \in \Omega$ and for each level layer $k \in[2, K]$ as :

$\mathrm{w}^{k}(\mathbf{s})^{(n)}=\frac{\beta \sum_{t_{i} \in \nu_{\mathbf{s}}} \mathrm{w}^{k}\left(t_{i}\right)^{(n-1)}-z^{k} a_{k} b_{k}-z_{k-1} c_{k} d_{k}}{\left|\nu_{\mathbf{s}}\right| * \beta+z^{k} b_{k}^{2}+z_{k-1} d_{k}^{2}}$ 
where

$$
\begin{aligned}
a_{k} & =e^{\tilde{\zeta}^{k}}\left(\left[\tilde{h}^{k} \nabla \tilde{\zeta}^{k}+\nabla \tilde{h}^{k}\right]^{T} \mathbf{v}^{\prime}+\tilde{h}^{k}\right)-\mathbb{I}_{C^{k}}\left(h^{k}\right) \\
& +g \Delta t f\left(\tilde{\zeta}^{k}, 0, \mathrm{w}^{k+1(n-1)}\right), \\
b_{k} & =g \Delta t f\left(\tilde{\zeta}^{k}, 1,0\right), \\
c_{k} & =e^{\tilde{\zeta}^{k-1}}\left(\left[\tilde{h}^{k-1} \nabla \tilde{\zeta}^{k-1}+\nabla \tilde{h}^{k-1}\right]^{T} \mathbf{v}^{\prime}+\tilde{h}^{k-1}\right)-\mathbb{I}_{C^{k-1}}\left(h^{k-1}\right) \\
& +g \Delta t f\left(\tilde{\zeta}^{k-1}, \mathrm{w}^{k-1(n-1)}, 0\right), \\
d_{k} & =g \Delta t f\left(\tilde{\zeta}^{k-1}, 0,1\right),
\end{aligned}
$$

and where the given boundary conditions on vertical winds $\left(\forall \mathbf{s} \in \Omega, \mathrm{w}^{1}(\mathbf{s})=\mathrm{w}^{K+1}(\mathbf{s})=0\right)$ hold. Note that vertical wind $\mathrm{w}^{k}$ is estimated considering variables related to the layer above the boundary $\left\{\mathrm{w}^{k+1}, h^{k}, \tilde{h}^{k}, v^{k}, z^{k}\right\}$ and the layer underneath the boundary $\left\{\mathrm{w}^{k-1}, h^{k-1}, \tilde{h}^{k-1}, v^{k-1}, z^{k-1}\right\}$. Finally, in a last step for each pixel locations and for each $k \in[1, K]$, the robust weights $z^{k}$ are in turn updated while variables $\left\{\mathbf{v}^{k}\right\}$ and $\left\{\mathrm{w}^{k}\right\}$ are kept fixed. The three previous minimization steps are iterated until a global convergence criterion is reached, that is to say until the variation of the estimated solution between two consecutive iterations becomes sufficiently small.

It is important to point out that the proposed 3D estimation methodology does not increase much the complexity of the original non-linear horizontal motion estimation problem. Indeed, given horizontal motion, the vertical wind estimation constitutes a linear quadratic problem which can be efficiently solved as presented in Eq 19.

\section{EXPERIMENTAL EVALUATION}

\section{A. Synthetic image sequence}

For an exhaustive evaluation, we have first relied on a simulated flow of an atmosphere decomposed into $K=3$ layers corresponding to low, medium and high clouds. The resulting synthetic images have been chosen to simulate a layered atmosphere that contains ascendant winds as a result of contraction at its base and expansion at its top. Let us describe the 3D motion simulation. A real cloud classification map (used in the next experiment) has been employed to dissociate the layers, and to assign them to different image regions $C^{k}$. Thus, for each layers $k$, a sparse image $h^{k}(t)$ of $128 \times 128$ pixels with a spatial resolution of $3 \times 3 \mathrm{~km}^{2}$ has been generated, representative of cloud pressure difference measurements on the assigned regions $C^{k}$ and of a fixed saturation value on the complementary domain. Textured image of mean $\bar{h}^{1}=200 \mathrm{hPa}, \bar{h}^{2}=200 \mathrm{hPa}$ and $\bar{h}^{3}=300 \mathrm{hPa}$ and with a standard deviation of 20 hecto Pascals (hPa) have been used to simulate cloud pressure difference values for the 3 layers. The three resulting images are presented in figure 2. Two different gaussian noises with variances respectively equal to $5 \%$ and $10 \%$ of the pressure difference amplitude have been generated to corrupted the previous textured images $h^{k}$. Three different image data sets have thus been generated. Consistent density values $\left(\bar{\rho}^{1}=1.22\right.$, $\bar{\rho}^{2}=1.00, \bar{\rho}^{3}=0.81$ and $\bar{\rho}^{4}=0.45$ ) have been chosen according to Eq. 14. An horizontal motion $v^{1}$ issued from a divergent sink has been imposed to the lower layer, while on the middle layer no horizontal winds $v^{2}=0$ has been considered. On the higher layer, a motion $v^{3}$ issued from a divergent source has been applied. The latter sink and source possess a decreasing influence while going away from the center of the image to its boundaries (motion amplitudes ranges in the interval $\sim 0-1.25$ pixel per frame, that is $\sim 0-4$ m.s. $\left.{ }^{-1}\right)$. Non-uniform vertical winds of strength $w^{2} \in[0.1,0.2]$ m.s. $^{-1}$ and $w^{3} \in[0.2,0.3]$ m.s. $^{-1}$ have been simulated on the boundaries shared respectively by the lower and the medium layers, and by the medium and the high layers respectively. The latter horizontal and vertical winds which are presented in figure 2 have been used to deform, according to the time integrated 3D-ICE model (Eq.15) and for each layer, the 3 different data sets (of increasing noise ratio). Thus, considering a time interval $\Delta t=900$ seconds, three sets of stack of images $\left[h^{1}(t), h^{2}(t), h^{3}(t)\right]$ were deformed to generate three different sets of propagated stack of images $\left[h^{1}(t+\Delta t), h^{2}(t+\Delta t), h^{3}(t+\Delta t)\right]$.

Horizontal and vertical winds retrieved with the 3D estimator using a multi-resolution approach for large displacements are presented in figure 2. For the three layer levels, vertical and horizontal winds are accurately estimated in cloudy regions. In observations free areas, vertical and horizontal winds appear to be consistent with the divergent and ascendant motions. Note that in the non cloudy regions, the estimator acts as a $3 \mathrm{D}$ wind extrapolator. Moreover, it can be noticed that the proposed layer interacting model significantly increases the estimation performances. In particular, the convergent motion of the lower layer is well characterized although only very few observations are available.

For comparison purpose we have run on this sequence the same estimator imposing a zero value to the unknown vertical components. This comes to use the $2 \mathrm{D}$ layered data model as proposed in [10]. As a result, this estimator calculates independent horizontal winds for the three different layers in the very same numerical implementation setup as for the $3 \mathrm{D}$ wind estimator. Results of this 2D layered estimation are presented in figure 2. It appears that the latter estimator completely fails to accurately characterize horizontal motion. This demonstrates that, although vertical wind $\left(\sim 0.1-0.3\right.$ m.s. $\left.{ }^{-1}\right)$ is weak compared to horizontal motion $\left(\sim 0-4 m_{. s} .^{-1}\right)$, its influence can not be neglected in the estimation process. A 3D data model clearly improves the results in such a situation.

The behavior of the 3D motion estimator accuracy has then been assessed using the synthetic observations generated for increasing noise levels. Let us denote by $|\Omega|$ the number of pixels in the discretized domain $\Omega$. The accuracy of horizontal wind estimates has been quantified using two criteria : the average absolute speed bias in pixels :

$$
\frac{1}{|\Omega|} \sum_{\mathbf{s}} \sqrt{\left(\left\|v^{k}\right\|-\left\|v_{\text {true }}^{k}\right\|\right)^{2}}
$$




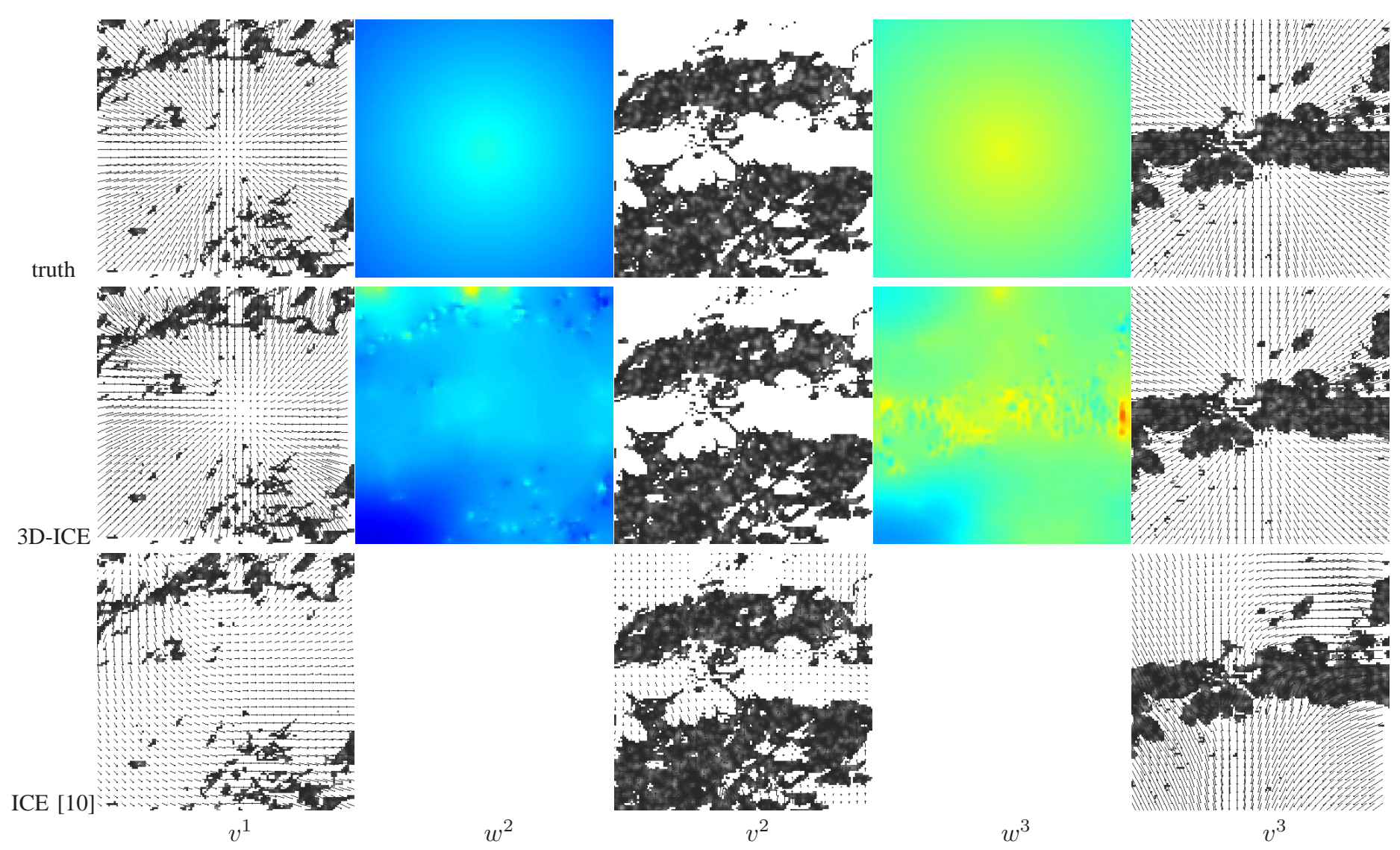

Fig. 2. Retrieval of horizontal motions and ascendant winds. Upper line : ground truth. Horizontal velocity fields $\left\{v^{1}, v^{2}, v^{3}\right\}$ used for simulation superimposed on cloud pressure difference images for a high (left), a medium (middle) and a low (right) layer. White areas correspond to regions where no data are available. Vertical wind fields $\left\{w^{2}, w^{3}\right\}$ used for simulation are displayed in-between the latter images (the legend bar associated to the color scale is displayed in figure 4). Middle line : comparison with 3D-ICE motion estimates. Horizontal winds estimated with the 3D-ICE model are superimposed on cloud pressure difference images for the high (left), the medium (middle) and the low (right) layer and estimated vertical wind maps $w^{3}$ and $w^{2}$ are displayed in-between the latter images. Bottom line : comparison with (2D) ICE motion estimates. Horizontal winds estimated with the ICE model are superimposed on cloud pressure difference images for the high (left), the medium (middle) and the low (right) layer.

and the average Barron's angular error in degrees [1] :

$$
\frac{1}{|\Omega|} \sum_{\mathrm{s}} \arccos \left(\frac{1+v^{k} \cdot v_{t r u e}^{k}}{\sqrt{1+\|\left. v^{k}\right|^{2}} \sqrt{1+\left\|v_{\text {true }}^{k}\right\|^{2}}}\right) \frac{180}{\pi},
$$

which is a criterion that accounts in the same time for angular and magnitude errors. Let us note that the speed bias is relevant only when Barron's angular error is not too important. The accuracy of vertical wind estimates has been quantified using the root mean square error in pixels :

$$
\sqrt{\frac{1}{|\Omega|} \sum_{\mathbf{s}}\left(w^{k}-w_{\text {true }}^{k}\right)^{2}} .
$$

In order to evaluate the influence of the new degree of freedom represented by vertical wind unknowns on the modeling (in particular on horizontal wind modeling), we also considered the average vertical motion norm :

$$
\frac{1}{|\Omega|} \sum_{\mathbf{s}}\left\|w^{k}\right\| .
$$

Results are presented in figure 3 and figure 4. For noise free observations, the 3D ICE model succeeds to estimate quite precisely horizontal motion (Barron's angular error and speed bias beneath 7 degrees and 0.1 pixel on the whole image) while the 2D data model fails to estimate correctly wind directions and speed (Barron's angular error and speed bias above 7 degrees and 0.1 pixel on the whole image). When the noise level is increased, horizontal motion estimation performance of the $3 \mathrm{D}$ and $2 \mathrm{D}$ estimator tend to be similar. Indeed, the estimation accuracy inevitably decreases as the 3D-ICE modeling is corrupted by noise, and the failure of the $2 \mathrm{D}$ estimator tends to reduce as the robust approach enables convergence towards the $2 \mathrm{D}$ solution in the presence of noise. Furthermore, for a sufficient noise level, the 3D and the $2 \mathrm{D}$ estimator prove to have similar performances. This behaviour can be explained by inspecting in figure 3 the decrease towards zero of the vertical motion norm mean with respect to the noise level, and the weakening of ascendant and descendant atmospheric activities in figure 4 .

Vertical wind estimation for noise free observations performs very good (root mean square error beneath $0.03 \mathrm{~ms}^{-1}$ ). Obviously, performances decrease when noise increases. However, because vertical motion norm tends towards zero for strong noise, there should exist an asymptotic limit for this error. 

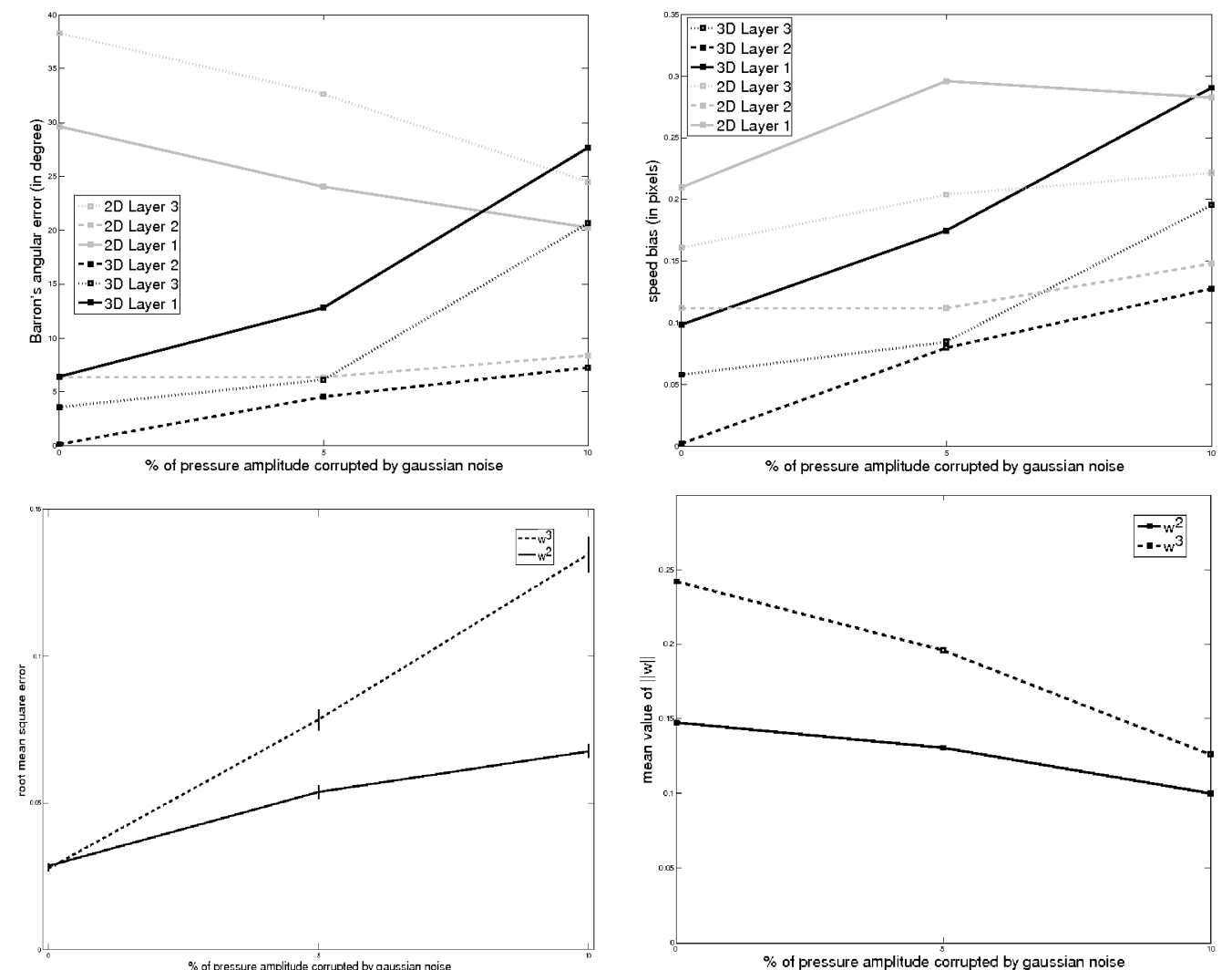

Fig. 3. Upper line : performance of the 3D-ICE and the (2D) ICE model for layered horizontal motion estimation. Barron's angular error (left) and speed bias (right) versus noise level. Bottom line : performance of the 3D-ICE model for vertical motion estimation. Root mean square error and mean value of the vertical component amplitude versus noise. Vertical bars represent standard deviations of square errors.

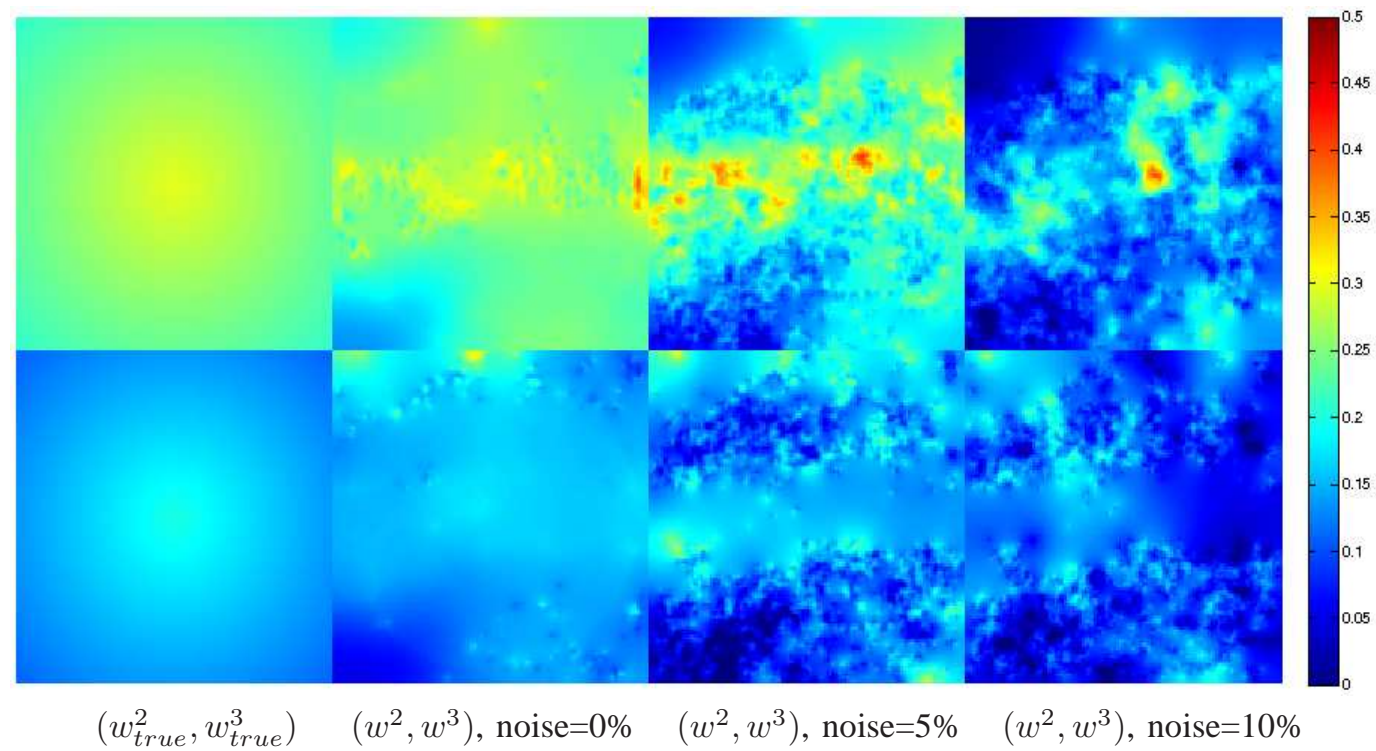

Fig. 4. Estimated vertical wind fields for increasing noise levels (vertical winds are expressed in m.s. ${ }^{-1}$ ).

It is however important to remark that the $3 \mathrm{D}$ estimates does not in any case deteriorate the estimation of the $2 \mathrm{D}$ horizontal wind fields. This can be checked on the second layer (which is the mayer with the biggest amount of data) and for which error estimates for the 2D and the 3D estimators stay acceptable whatever the noise level. Besides, for a moderated noise level, the $3 \mathrm{D}$ model outperforms systematically the $2 \mathrm{D}$ model.

\section{B. METEOSAT satellite image sequences}

We then turned to qualitative evaluations on METEOSAT Second Generation (MSG) meteorological image sequences acquired at a rate of an image every 15 minutes. This 


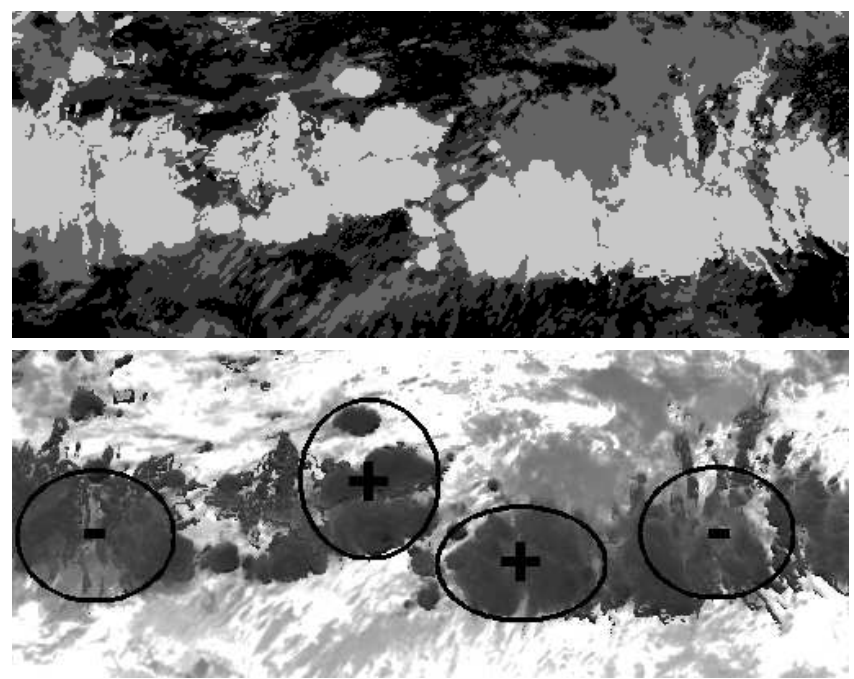

Fig. 5. Top of cloud classification and pressure image (over the Gulf Guinea the 5-June-2004 at 13h30). Above : EUMETSAT cloud top classification into 3 coarse layers : high, middle and low displayed respectively in white light and dark gray. Black regions correspond to missing observations. Below : EUMETSAT top of cloud pressure image. Plausible scenarios for vertical motion which have been represented by positive and negative symbols are superimposed on the image. White regions correspond to missing observations.

benchmark data, which has been provided by the EUMETSAT consortium, is composed of images of top of cloud pressure and cloud-classification images. The image spatial resolution is $3 \times 3 \mathrm{~km}^{2}$ at the center of the whole Earth image disk. The cloud-classifications were used to segment images into 3 broad layers, at low, intermediate and high altitude ${ }^{1}$. Applying the methodology described in section III-C, pressure difference images for the 3 layers were derived from pressure images.

The first sequence chosen for evaluation was composed of top of cloud pressure images covering an area over the Gulf Guinea, during part of one day (5-June-2004) from $13 \mathrm{~h} 30$ to $14 \mathrm{~h} 15$ UTC (universal time, similar to GMT : Greenwich mean time). The first image of the sequence is displayed together with the related cloud classification in figure 5 . Figure 6 displays the 4 consecutive images of 512 by 200 pixels (covering an area of about 1500 x $600 \mathrm{~km}^{2}$ ) related to the higher layer pressure difference, together with $3 \mathrm{D}$ estimated wind fields.

One can visualize large convective systems for the higher layer. They are characterized by a strong ascendant flow which is smoothly reversed after reaching the tropopause cover. Such scenarios, which are represented in figure 5, have been correctly estimated. Estimated winds are displayed in figure 6. Note that a multi-resolution approach was here maintained in order to assess the temporal consistency of vertical wind estimates.

\footnotetext{
${ }^{1}$ We note that the EUMETSAT extraction procedure was not correctly tuned when the classification was extracted (June 2004). After comparison with cloud classifications obtained by other methods, it appeared that general cloud coverage was under evaluated. The high-level cloud coverage were also found to be underestimated in favor of medium-level clouds. These differences should not change radically the following evaluation, since a large majority of cloudy pixels are correctly assigned. Nevertheless, better classification products are likely to significantly enhance the performance of the method.
}

The second sequence chosen for evaluation is the same sequence of top of cloud pressure images used to evaluate the 2D layered wind estimator in [10]. It is constituted by 5 images of $512 \times 512$ pixels covering an area over the north Atlantic Ocean (of about $1500 \times 1500 \mathrm{~km}^{2}$ ), off the Iberian peninsula, during part of one day (5-June-2004), from $12 \mathrm{~h} 00$ to $13 \mathrm{~h} 00 \mathrm{UTC}$. The first image of the sequence is displayed together with the related cloud classification in figure 7 . The sequence of pressure difference images related to the 3 layers are presented in figure 8 together with the estimated horizontal wind fields. In order to increase the large displacement estimation accuracy, we have replaced the multi-resolution approach by the two-stage estimation scheme proposed in [10].

Estimated horizontal wind fields appeared to be visually consistent and in agreement with previous results in [10]. By a careful visual inspection of the sequence, meteorologists have put forwards some plausible scenarios concerning vertical winds which are represented in figure 7. In particular, the eastwards front visible on the highest layer (and located on the bottom left side of the images) shoud be preceded by descendant motion, which has been well characterized in $w^{3}$ maps of figure 9. Ascendant winds should be related to small convective systems, which can be observed in isolated cloudy regions of the highest layer (located mainly on the right side of the images). These phenomena have correctly been characterized in $w^{3}$ maps of figure 9. The large cloud system of the middle layer (located in the middle, on the left side of the images) is likely to be associated to southwards descendant winds $w^{2}$ and northwards ascendant winds $w^{3}$. The big vortex structure of the lower layer in the image center and the small vortex in the image upper part which corresponds to clouds of both, the lower and the middle layers, are likely to be associated to ascendant winds $w^{2}$. 


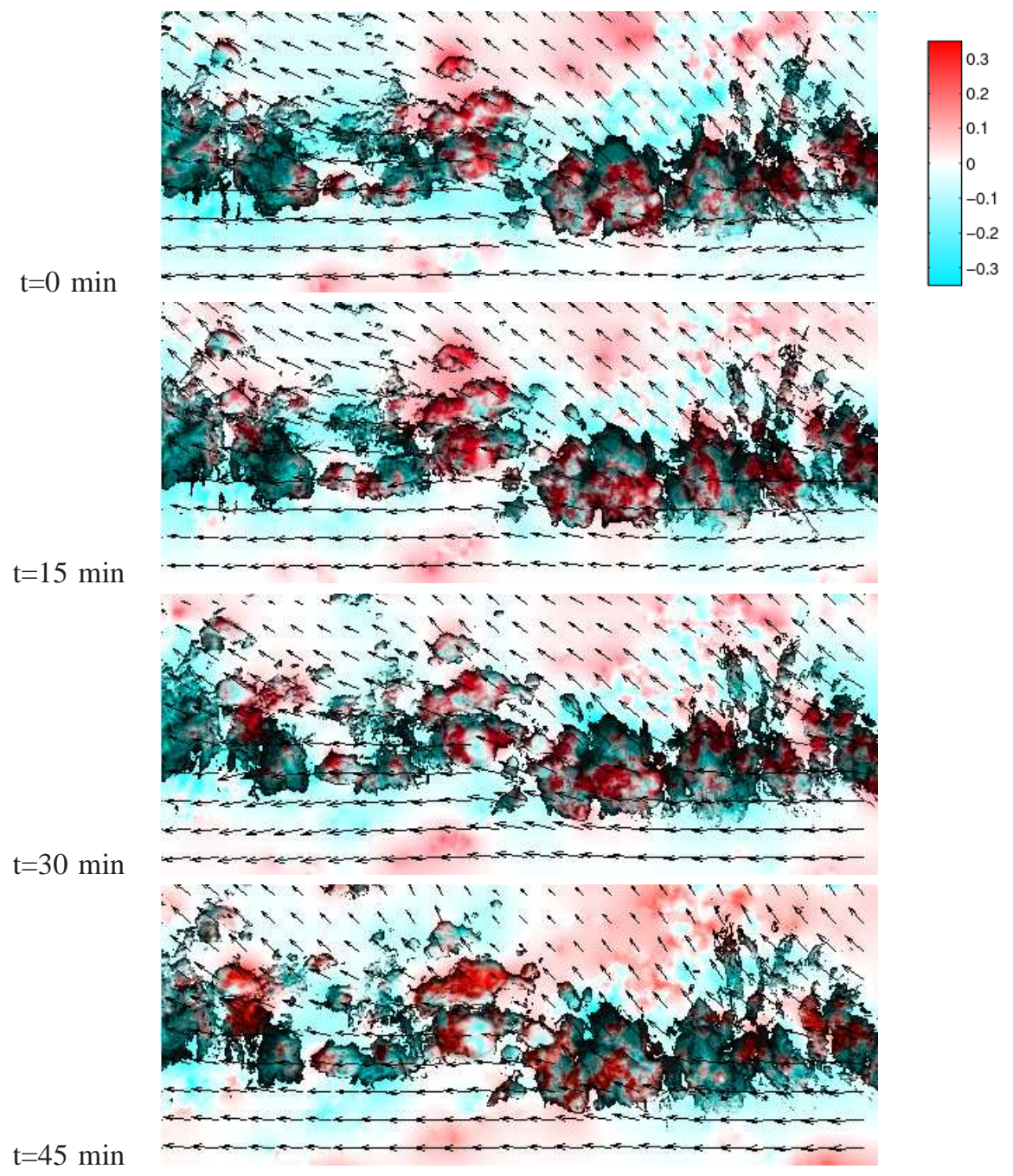

Fig. 6. Estimation of 3D wind in atmospheric convective systems (over the Gulf Guinea the 5-June-2004 from 13 h30 to 14 h15 UTC). Cloud pressure difference images of the highest layer at 4 consecutive times. Estimated horizontal wind vectors which have been superimposed on the images range in the interval $[0,10] \mathrm{m.s.}^{-1}$. Retrieved vertical wind maps on the highest layer lower boundary have been superimposed on the pressure difference images. Vertical winds range in the interval $[-0.5,0.5]$ m.s. $\left.{ }^{-1}\right)$.
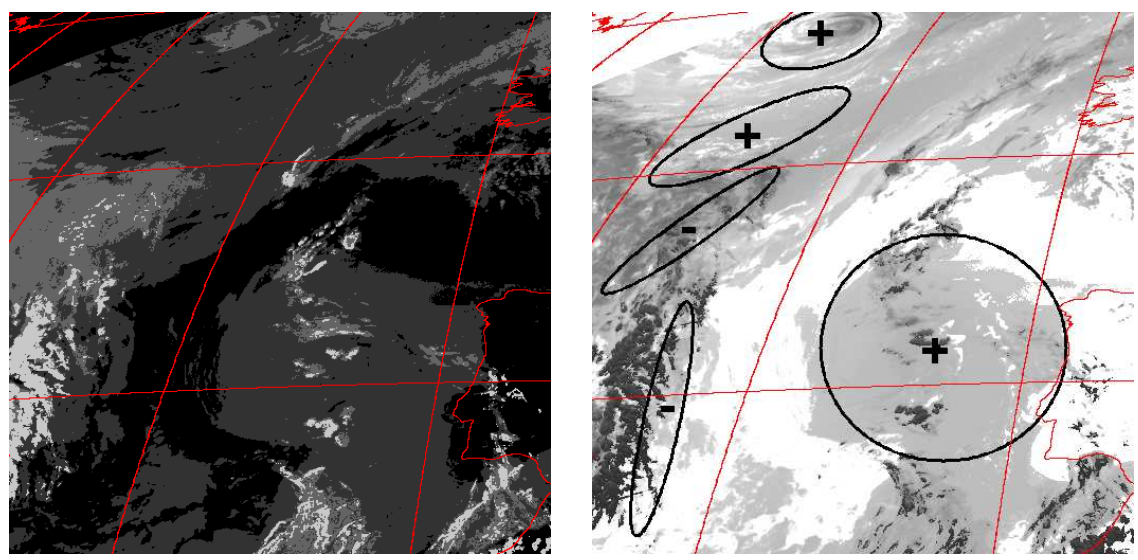

Fig. 7. Top of cloud pressure image and classification (over the north Atlantic Ocean the 5-June-2004 at 12h00). On the left : EUMETSAT cloud top classification into 3 coarse layers : high, middle and low displayed respectively in white light and dark gray. Black regions correspond to missing observations and red lines represent costal contours, meridians and parallels (every $10^{\circ}$ ). Black regions correspond to missing observations On the right : EUMETSAT top of cloud pressure image. Plausible scenarios for vertical motion which have been represented by positive and negative symbols are superimposed on the image. White regions correspond to missing observations. 
Estimated vertical wind fields of figure 9 seem only up to some extent in agreement with the two previous scenarios. However, these behaviors are only probable global scenarios which constitute very coarse approximations of the actual atmospheric dynamics.

Furthermore, let us remark that the time consistency of the first and the second image sequences, together with the correct range of estimated winds, is a testimony of the stability of the 3D estimation method.

\section{CONCLUSiOnS}

In this paper, we have presented a motion estimation method solving for the first time the complex problem of 3D winds field recovery from satellite image sequences. In order to manage incomplete observations, physical knowledge on 3D mass exchanges between atmospheric layers have been introduced within an optical flow scheme.

The estimator is based on a functional minimization. The data term relies on the 3D-ICE model which describes the dynamics of an interacting stack of atmospheric layers. The 3D-ICE model applies on a set of sparse pressure difference images related to the different atmospheric layers. A method is proposed to reconstruct such observations from satellite top of cloud pressure images and classification maps. To overcome the problem of sparse observations, a robust estimator is introduced in the data term. The data term is combined with a smoother that preserves the bi-dimensional divergent and vorticity structures of the three-dimensional flow and reinforces regions of homogeneous vertical winds.

An evaluation first performed on a synthetic image sequence, and latter on 2 METEOSAT infrared image sequences demonstrate the stability and the efficiency of the method even in the difficult case of noisy and very sparse image observations.

\section{APPENDIX I : VERTICAL INTEGRATION OF THE} CONTINUITY EQUATION USING THE ISOBARIC COORDINATE SYSTEM

For compressible fluids, the continuity equation in the $(x, y, p)$ coordinates system reads:

$$
-\frac{\partial \omega}{\partial p}=\left(\frac{\partial u}{\partial x}+\frac{\partial v}{\partial y}\right)_{p} .
$$

We denote by $\mathbf{v}=(u, v)$ the horizontal velocity and by $s^{k}$ and $s^{k+1}$ the altimetric surfaces with $p\left(s^{k}\right)>p\left(s^{k+1}\right)$. Integrating Eq.24 in the pressure interval $\left[p\left(s^{k+1}\right), p\left(s^{k}\right)\right]$ yields to

$$
\begin{aligned}
{[\omega]_{s^{k}}^{s^{k+1}} } & =\left.\operatorname{div}\right|_{p} \int_{p\left(s^{k+1}\right)}^{p\left(s^{k}\right)} \mathbf{v} d p-\left.\mathbf{v}\left(s^{k}\right) \cdot \nabla_{x y} p\left(s^{k}\right)\right|_{s^{k}} \\
& +\left.\mathbf{v}\left(s^{k+1}\right) \cdot \nabla_{x y} p\left(s^{k+1}\right)\right|_{s^{k+1}} .
\end{aligned}
$$

For a better understanding, let us note that to obtain Eq. 25, we have used the Leibnitz formula to perform the vertical integration of Eq. 24 in the pressure interval $\left[p\left(\mathbf{x}, s^{k+1}\right), p\left(\mathbf{x}, s^{k}\right)\right]$ varying with coordinates $\mathbf{x}=(x, y)$. This formula, which is valid for all integrable and derivable function $f(\mathbf{x}, p)$ and for all interval $[a(\mathbf{x}), b(\mathbf{x})]$ with boundaries varying with $\mathbf{x}$, reads in $1 \mathrm{D}$ :

$$
\begin{array}{r}
\int_{a(\mathbf{x})}^{b(\mathbf{x})} \frac{\partial f(\mathbf{x}, p)}{\partial \mathbf{x}} d p=\frac{\partial}{\partial \mathbf{x}}\left(\int_{a(\mathbf{x})}^{b(\mathbf{x})} f(\mathbf{x}, p) d p\right) \\
-f(\mathbf{x}, b(\mathbf{x})) \frac{\partial b(\mathbf{x})}{\partial \mathbf{x}}+f(\mathbf{x}, a(\mathbf{x})) \frac{\partial a(\mathbf{x})}{\partial \mathbf{x}} .
\end{array}
$$

Note that in Eq. 25 the divergence operator $\left.\operatorname{div}\right|_{p}$ is defined in isobaric coordinates. Thus, in this Eq. 25 divergence is computed at point $\mathrm{x}$ in a constant pressure interval.

Moreover, expanding $\omega$ in the $(x, y, z)$ coordinates system and using the hydrostatic assumption $\left(\frac{\partial p}{\partial z}=-\rho g\right)$ yields to

$$
\omega=\frac{d p}{d t}=\frac{\partial p}{\partial t}+\mathbf{v} \cdot \nabla_{x y}(p)-\mathrm{w} \rho g
$$

where partial derivative operators and the vertical velocity $\mathrm{w}$ are in $z$ coordinates and where we have introduced the density functions $\rho$ and the gravity constant $g$. Assuming that pressure partial derivatives computed on the surface $s^{k}$ or at constant height are similar, that is to say assuming that $s^{k}$ is flat in the vicinity of a pixel, we can derive the following approximations:

$$
\begin{aligned}
\left.\mathbf{v}\left(s^{k}\right) \cdot \nabla_{x y} p\left(s^{k}\right)\right|_{s^{k}} & \simeq \mathbf{v}\left(s^{k}\right) \cdot \nabla_{x y} p\left(s^{k}\right) \\
\left.\frac{\partial p\left(s^{k}\right)}{\partial t}\right|_{s^{k}} & \simeq \frac{\partial p\left(s^{k}\right)}{\partial t} .
\end{aligned}
$$

Thus spatial gradients terms reduce to zero when merging Eq. 25 and Eq. 27, and we obtain

$g[\rho \mathrm{w}]_{s^{k+1}}^{s^{k}}+\left.\left.\frac{\partial\left(p\left(s^{k+1}\right)-p\left(s^{k}\right)\right)}{\partial t}\right|_{I_{s}} \simeq \operatorname{div}\right|_{p} \int_{p\left(s^{k+1}\right)}^{p\left(s^{k}\right)} \mathbf{v} d p$

where we have denoted by $I_{s}$ the altimetric interval between surfaces $s^{k}$ and $s^{k+1}$. Let us now define the following quantities :

$$
\begin{aligned}
\delta p^{k} & =p\left(s^{k}\right)-p\left(s^{k+1}\right) \\
\mathbf{v}^{k} & =\frac{1}{\delta p^{k}} \int_{p\left(s^{k+1}\right)}^{p\left(s^{k}\right)} \mathbf{v} d p
\end{aligned}
$$

We can then rewrite Eq. 29 as

$$
g \rho\left(s^{k}\right) \mathrm{w}\left(s^{k}\right)-\left.g \rho\left(s^{k+1}\right) \mathrm{w}\left(s^{k+1}\right) \simeq \frac{\partial \delta p^{k}}{\partial t}\right|_{I_{s}}+\left.\operatorname{div}\left(\delta p^{k} \mathbf{v}^{k}\right)\right|_{p}
$$

The approximation $\left.\left.\operatorname{div}\left(\mathbf{v}^{k}\right)\right|_{p} \simeq \operatorname{div}\left(\mathbf{v}^{k}\right)\right|_{I_{s}}$ is relevant since we are considering the divergence of averaged horizontal winds which are characterized by very small vertical fluctuations (compared to horizontal fluctuations). Therefore one should obtain very similar divergence measurements for horizontal winds averaged in a constant pressure interval or for horizontal winds averaged in a varying altimetric interval $I_{s}$ (or pressure interval $\left[p\left(s^{k+1}\right), p\left(s^{k}\right)\right]$ ). Thus, we can approximate the latter equation by

$$
\begin{array}{r}
g \rho\left(s^{k}\right) \mathrm{w}\left(s^{k}\right)-g \rho\left(s^{k+1}\right) \mathrm{w}\left(s^{k+1}\right) \simeq \\
\left.\frac{\partial \delta p^{k}}{\partial t}\right|_{I_{s}}+\left.\mathbf{v}^{k} \cdot \nabla_{x y}\left(\delta p^{k}\right)\right|_{I_{s}}+\left.\delta p^{k} \operatorname{div}\left(\mathbf{v}^{k}\right)\right|_{I_{s}},
\end{array}
$$


Simplifying notations of operators defined for the altimetric interval $I_{s}$, we obtain the relation :

$g \rho\left(s^{k}\right) \mathrm{w}\left(s^{k}\right)-g \rho\left(s^{k+1}\right) \mathrm{w}\left(s^{k+1}\right) \simeq \frac{d \delta p^{k}}{d t}+\delta p^{k} \operatorname{div}\left(\mathbf{v}^{k}\right)$,

which consitutes a proper image-adapted model for observations $\delta p^{k}$ related to a layer defined in the interval $I_{s}$.

\section{APPENDIX II : TEMPORAL INTEGRATION OF THE 3D ICE MODEL}

The 3D ICE model reads:

$$
\frac{d h^{k}(\mathbf{s}, t)}{d t}+h^{k}(\mathbf{s}, t) \nabla \cdot \mathbf{v}^{k}=g\left(\bar{\rho}^{k} \mathbf{w}^{k}-\bar{\rho}^{k+1} \mathbf{w}^{k+1}\right) \text {, }
$$

Assuming a constant velocity over the lapse of time $\Delta t$, we have here a first order ordinary differential equation of the form $y^{\prime}=m y+p$ that can be integrated. If $\operatorname{divv}^{k} \neq 0$, the general solution of this differential equation is :

$$
h^{k}(\mathbf{s}, t)=\nu e^{-\left(\operatorname{div} \mathbf{v}^{k}\right) t}+g \frac{\bar{\rho}^{k} \mathrm{~W}^{k}-\bar{\rho}^{k+1} \mathrm{~W}^{k+1}}{\operatorname{div} \mathbf{v}^{k}}
$$

Expressing the previous equation at time $t=0$, we obtain the constant $\nu$ :

$$
\nu=h^{k}(\mathbf{s}, t)-g \frac{\bar{\rho}^{k} \mathrm{w}^{k}-\bar{\rho}^{k+1} \mathrm{w}^{k+1}}{\operatorname{div} \mathbf{v}^{k}},
$$

and by expressing the same equation at time $t=t+\Delta t$ we obtain

$$
\begin{aligned}
& h^{k}\left(\mathbf{s}+\mathbf{v}^{k}, t+\Delta t\right) e^{\operatorname{div} \mathbf{v}^{k}}-h^{k}(\mathbf{s}, t),= \\
& g \Delta t \frac{\bar{\rho}^{k} \mathbf{w}^{k}-\bar{\rho}^{k+1} \mathbf{w}^{k+1}}{\operatorname{div} \mathbf{v}^{k}}\left(e^{\operatorname{div} \mathbf{v}^{k}}-1\right),
\end{aligned}
$$

where $\Delta t$ denotes the time interval expressed in seconds between two consecutive images and where horizontal divergence $\operatorname{divv}^{k}$ are expressed in $\mathrm{f}^{-1}$, with $\mathrm{f}$ denoting number of frames. For the particular case $\operatorname{divv}^{k}=0$, the latter equation becomes :

$$
\begin{aligned}
& h^{k}\left(\mathbf{s}+\mathbf{v}^{k}, t+\Delta t\right)-h^{k}(\mathbf{s}, t), \quad= \\
& -g \Delta t\left(\bar{\rho}^{k} \mathbf{w}^{k}-\bar{\rho}^{k+1} \mathbf{w}^{k+1}\right) .
\end{aligned}
$$

\section{REFERENCES}

[1] J. Barron, D. Fleet, and S. Beauchemin. Performance of optical flow techniques. Int. J. Computer Vision, 12(1):43-77, 1994.

[2] T. Bellerby. High-resolution 2-d cloud-top advection from geostationary satellite imagery. IEEE transactions on geoscience and remote sensing, 44(12):3639-3648, 2006.

[3] J. Bergen, P. Burt, R. Hingorani, and S. Peleg. A three-frame algorithm for estimating two-component image motion. IEEE Trans. Pattern Anal. Machine Intell., 14(9):886-895, Sept. 1992.

[4] M. Black and P. Anandan. The robust estimation of multiple motions: Parametric and piecewise-smooth flow fields. Computer Vision and Image Understanding, 63(1):75-104, 1996.

[5] T. Corpetti, E. Mémin, and P. Pérez. Dense estimation of fluid flows. IEEE Trans. Pattern Anal. Machine Intell., 24(3):365-380, 2002.

[6] A. Cuzol and E. Memin. Vortex and source particles for fluid motion estimation. In Proc. 5th Int. Conf. on Scale-Space and PDE methods in Computer Vision (Scale-Space'05), Hofgeismar, Germany, 2005.

[7] A. de Saint-Venant. Theorie du mouvement non-permanent des eaux, avec application aux crues des rivieres et l'introduction des marees dans leur lit. C. R. Acad. Sc. Paris, 73:147-154, 1871.

[8] J. Fitzpatrick. The existence of geometrical density-image transformations corresponding to object motion. Comput. Vision, Graphics, Image Proc., 44(2):155-174, Nov. 1988.
[9] P. Gamba. Meteorological structures shape description and tracking by means of bi-rme matching. IEEE trans. on Geoscience and Remote sensing, 37(2):1151-1161, Mar. 1999.

[10] P. Heas, E. Memin, N. Papadakis, and A. Szantai. Layered estimation of atmospheric mesoscale dynamics from satellite imagery. IEEE trans. on Geoscience and Remote sensing, 45(12):4087-4104, 2007.

[11] P. Holland and R. Welsch. Robust regression using iteratively reweighted least-squares. Commun. Statis.-Theor. Meth., A6(9):813-827, 1977.

[12] J. Holton. An introduction to dynamic meteorology. Academic press, 1992.

[13] B. Horn and B. Schunck. Determining optical flow. Artificial Intelligence, 17:185-203, 1981.

[14] R. Larsen, K. Conradsen, and B. Ersboll. Estimation of dense image flow fields in fluids. IEEE trans. on Geoscience and Remote sensing, 36(1):256-264, Jan. 1998.

[15] J. Leese, C. Novack, and B. Clark. An automated technique for obtained cloud motion from geosynchronous satellite data using cross correlation. Journal of applied meteorology, 10:118-132, 1971.

[16] H. Lutz. Cloud processing for meteosat second generation. Technical report, European Organisation for the Exploitation of Meteorological Satellites (EUMETSAT), Available at : http://www.eumetsat.de, 1999.

[17] E. Mémin and P. Pérez. Fluid motion recovery by coupling dense and parametric motion fields. In Int. Conf. on Computer, ICCV'99, pages 620-625, 1999.

[18] W. Menzel, W. Smith, and T. Stewart. Improved cloud motion wind vector and altitude assignment using vas. Journal of Climate and Applied meteorology, 22(3):377-384, 1983.

[19] A. Ottenbacher, M. Tomasini, K. Holmund, and J. Schmetz. Lowlevel cloud motion winds from Meteosat high-resolution visible imagery. Weather and Forecasting, 12(1):175-184, March 1997.

[20] J. Schmetz, K. Holmund, J. Hoffman, B. Strauss, B. Mason, V. Gaertner, A. Koch, and L. V. D. Berg. Operational cloud-motion winds from meteosat infrared images. Journal of Applied Meteorology, 32(7):1206$1225,1993$.

[21] D. Suter. Motion estimation and vector splines. In Proc. Conf. Comp. Vision Pattern Rec., pages 939-942, Seattle, USA, June 1994.

[22] A. Szantai and F. Desalmand. Using multiple channels from MSG to improve atmospheric motion wind selection and quality. In 7 th International Winds Workshop, EUMETSAT EUM P 42, pages 307-314, Helsinki, Finland, June 2004.

[23] J. Yuan, C. Schnoerr, and E. Memin. Discrete orthogonal decomposition and variational fluid flow estimation. Journ. of Mathematical Imaging and Vison, (accepted for publication), 2006.

[24] L. Zhou, C. Kambhamettu, and D. Goldgof. Fluid structure and motion analysis from multi-spectrum $2 \mathrm{~d}$ cloud images sequences. In Proc. Conf. Comp. Vision Pattern Rec., volume 2, pages 744-751, USA, 2000. 


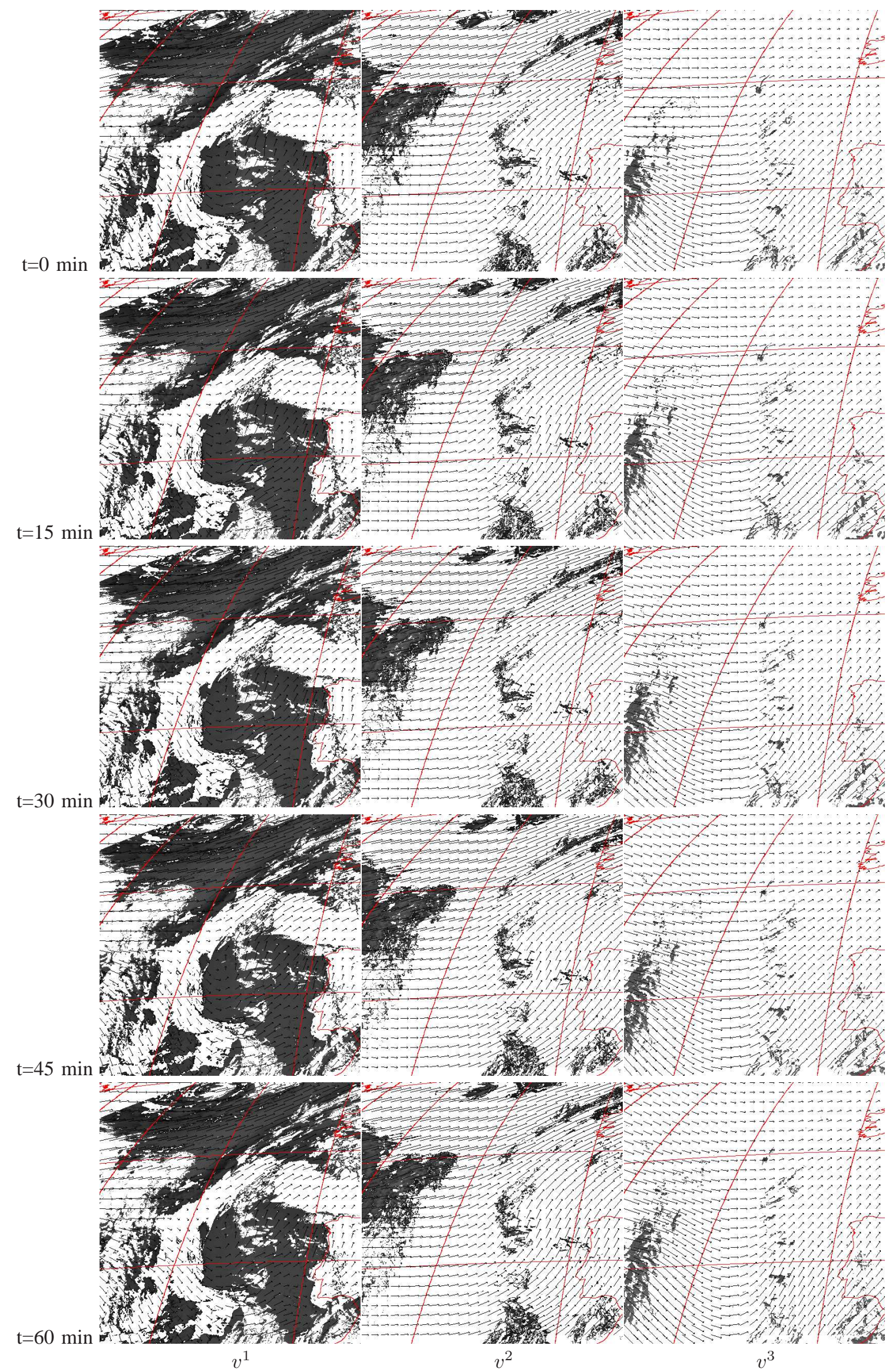

Fig. 8. Estimation of $3 D$ wind in a 3-layer atmosphere : horizontal winds. Horizontal winds $\left(v^{1}, v^{2}, v^{3}\right)$ in correspondence to cloud pressure difference images (over the north Atlantic Ocean the 5-June-2004 from $12 \mathrm{~h} 00$ to $13 \mathrm{~h} 00$ UTC) related to the high (left row), the middle (middle row) and the low (right row) layers at consecutive times. Estimated horizontal wind vectors range in the interval of $[0,15] \mathrm{m.s} .^{-1}$. 

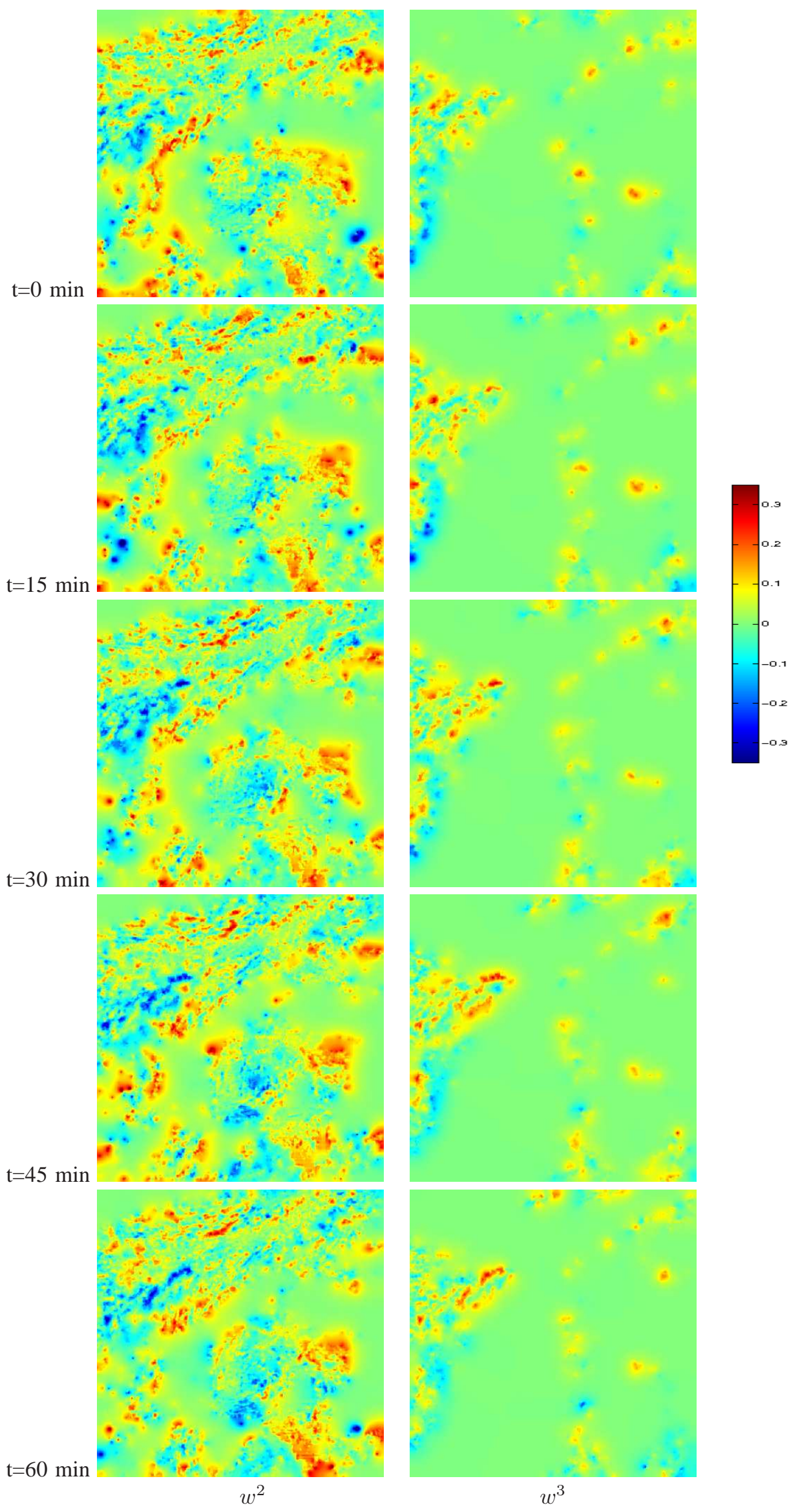

Fig. 9. Estimation of $3 \mathbf{D}$ wind in a 3-layer atmosphere : vertical winds. Vertical winds $\left(w^{2}\right.$, $\left.w^{3}\right)$ (over the north Atlantic Ocean the 5-June-2004 from $12 \mathrm{~h} 00$ to $13 \mathrm{hOO}$ UTC) at consecutive times. Estimated vertical winds range in the interval $[-0.2,0.4] \mathrm{m.s}^{-1}$. 